\title{
ON JULIA DIRECTIONS OF ENTIRE FUNCTIONS OF SMALL ORDER
}

\author{
JIN LU*
}

\begin{abstract}
In this paper, the relationship between Julia direction and the growth of entire function of order $0<\lambda \leq 1 / 2$ is discussed.
\end{abstract}

\section{Introduction}

A ray $\chi(\theta)=\left\{z=r e^{i \theta}: 0<r<+\infty\right\}$ in the complex plane is called a Julia direction of entire function $f(z)$, if $f(z)$ takes all finite complex numbers infinitely often in any sector containing $\chi(\theta)$, with at most one finite exceptional value. So in any sector containing Julia direction $\chi(\theta)$ of $f(z)$, the set of roots of equation $f(z)=\alpha$ is unbounded if $\alpha$ is not such a exceptional value, hence $f(z)$ can not tend to $\infty$ as $|z| \rightarrow+\infty$ in the sector. But if a ray $\chi(\theta)$ is not a Julia direction of $f(z)$, what will happen about $f(z)$ ? In [2] H. Yoshida conjectured: If $f(z)$ is an entire function of order less than $1 / 2$ and $\chi(\theta)$ is ray, then either $\chi(\theta)$ is a Julia direction of $f(z)$ or $f(z)$ tends $\infty$ as $|z| \rightarrow+\infty$ in some sector containing $\chi(\theta)$. In this paper we discuss this question and prove that $\mathrm{H}$. Yoshida's conjecture is affirmative under some conditions. These conditions are different from the conditions which were discussed in [2] and we also give a estimate about the growth of this kind of functions in a sector that does not contain Julia directions. Our main result is:

THEOREM 1. Let $f(z)$ be an entire function of order $0<\lambda \leq 1 / 2$, and $f(z)$ be regular growth, i.e., its lower order is equal to its order, then either $\chi(\theta)$ is a Julia direction of $f(z)$ or in some sector $G$ containing $\chi(\theta)$,

holds.

$$
\lim _{\substack{|z| \rightarrow+\infty \\ z \in G}} \frac{\log \log |f(z)|}{\log |z|}=\lambda
$$

In Section 3 of this paper, an interesting corollary of this theorem will be discussed.

\footnotetext{
* Project supported by Chinese NNSF.

2000 Mathematics Subject Classification: Primary 32D35, 30D20.

Received June 4, 2001; revised November 19, 2001.
} 


\section{Notations and lemmas}

Lemma 1 ([1, Theorem 3]). Let $f(z)$ be an entire function of lower order $0<\mu \leq 1 / 2$, then there exists an asymptotic path $L$ tending to $\infty$, such that

$$
\liminf _{\substack{|z| \rightarrow+\infty \\ z \in L}} \frac{\log \log |f(z)|}{\log |z|}=\mu .
$$

Lemma 2 ([5] or [6, Lemma 6.6]). Let $f(z)$ be a meromorphic function on the disc $|z| \leq R$. Set

$$
N=n(R, f=0)+n(R, f=1)+n(R, f=\infty),
$$

where $n(R, f=a)$ denotes the number of roots of the equation $f(z)=a$ on $|z| \leq R$ (Counting multiplicity). If the distance from $z=0$ to these $N$ points has a positive lower bound $d$, then there exists a constant $C$ such that for any $0<r<R$,

$$
T(r, f)<\frac{C R(N+1)}{R-r} \log \frac{2 R^{2}(N+1)}{d(R-r)}+\log ^{+}|f(0)|,
$$

where $T(r, f)$ denotes the characteristic function of $f(z)$.

Denote $\Omega\left(\theta_{1}, \theta_{2}\right)=\left\{z=r e^{i \theta}: \theta_{1} \leq \theta \leq \theta_{2}, 0 \leq r<+\infty\right\}$ and $\Omega\left(\theta_{1}, \theta_{2}, r_{1}, r_{2}\right)=$ $\left\{z=r e^{i \theta}: \theta_{1} \leq \theta \leq \theta_{2}, r_{1} \leq r \leq r_{2}\right\}$.

LEMMA 3. Let $f(z)$ be an entire function, which assumes distinct complex numbers $\alpha$ and $\beta$ finite times in the sector $\Omega(-\sigma, \sigma)(\sigma>0)$. If there exists a sequence $\left\{z_{n}\right\} \quad\left(\left|z_{n}\right| \rightarrow \infty\right)$ in $\Omega(-\sigma / 128, \sigma / 128)$ such that

$$
\left|f\left(z_{n}\right)\right|<K(n),
$$

then there exists a constant $C$ which is independent of $n$ and $K(n)$ such that for each $n$,

$$
\log |f(z)| \leq C(\log K(n)+1)
$$

holds on the set $E_{n}=\left\{z=\left|z_{n}\right| e^{i \theta}: \theta \in[-\sigma / 128, \sigma / 128]\right\}$.

Proof. It suffices to prove the conclusion holds when $n$ is sufficiently large. Consider the disc $D_{n}=\left\{z:\left|z-z_{n}\right| \leq\left|z_{n}\right| \sin (\sigma / 16)\right\}$. It is easy to see that $E_{n} \subset D_{n}$ and $D_{n} \subset \Omega(-\sigma, \sigma)$. Since $\left|z-z_{n}\right| \leq\left|z_{n}\right| \sin (\sigma / 32)$ holds for any $z \in E_{n}$, from Possion-Jensen formula we have for any $z \in E_{n}$,

$$
\begin{aligned}
\log |f(z)| & \leq \frac{\left|z_{n}\right| \sin (\sigma / 16)+\left|z_{n}\right| \sin (\sigma / 32)}{\left|z_{n}\right| \sin (\sigma / 16)-\left|z_{n}\right| \sin (\sigma / 32)} m\left(\left|z_{n}\right| \sin \frac{\sigma}{16}, z_{n}, f\right) \\
& \leq C T\left(\left|z_{n}\right| \sin \frac{\sigma}{16}, z_{n}, f\right) \\
& \leq C\left\{T\left(\left|z_{n}\right| \sin \frac{\sigma}{16}, z_{n}, \frac{f-\alpha}{\beta-\alpha}\right)+1\right\}
\end{aligned}
$$


where $T\left(r, z_{0}, f\right)$ denotes the characteristic function of $f(z)$ on the disc $\left\{z:\left|z-z_{0}\right| \leq r\right\} \quad$ (In this paper $C$ is always a constant, although it maybe changes in each step).

Let $N$ be the number of times that $f(z)$ assumes $\alpha$ and $\beta$ in $\Omega(-\sigma, \sigma)$. When $n$ is sufficiently large, the distance from $z_{n}$ to these $N$ points is larger than $\left|z_{n}\right| \sin (\sigma / 16)$. Noticing that the disc $\left\{z:\left|z-z_{n}\right| \leq\left|z_{n}\right| \sin (\sigma / 8)\right\}$ lies also in $\Omega(-\sigma, \sigma)$, from Lemma 2 we have

$$
\begin{aligned}
T\left(\left|z_{n}\right| \sin \frac{\sigma}{16}, z_{n}, \frac{f-\alpha}{\beta-\alpha}\right) \leq & C\left\{\frac{\left|z_{n}\right| \sin (\sigma / 8)(N+1)}{\left|z_{n}\right| \sin (\sigma / 8)-\left|z_{n}\right| \sin (\sigma / 16)}\right. \\
& \times \log \frac{2\left(\left|z_{n}\right| \sin (\sigma / 8)\right)^{2}(N+1)}{\left(\left|z_{n}\right| \sin (\sigma / 8)-\left|z_{n}\right| \sin (\sigma / 16)\right)\left|z_{n}\right| \sin (\sigma / 16)} \\
& \left.\quad+\log ^{+}\left|\frac{f\left(z_{n}\right)-\alpha}{\beta-\alpha}\right|+1\right\} \\
\leq & C(\log K(n)+1) .
\end{aligned}
$$

Combining these two estimates the conclusion is obtained.

Denote $M\left(\Omega\left(-\theta_{1}, \theta_{2}, r_{1}, r_{2}\right), f\right)=\max _{z \in \Omega\left(-\theta_{1}, \theta_{2}, r_{1}, r_{2}\right)}|f(z)|, \quad$ and $\quad M(r, f)=$ $\max _{|z| \leq r}|f(z)|$.

LEMMA 4. Let $f(z)$ be an entire function, which assumes distinct complex numbers $\alpha$ and $\beta$ finite times in the sector $\Omega(-2 \sigma, 2 \sigma)(\sigma>0)$. If there exists a sequence $\left\{z_{n}\right\}\left(\left|z_{n}\right| \rightarrow \infty\right)$ such that

$$
\left|f\left(\left|z_{n}\right|\right)\right|<K(n) .
$$

Then for any fixed $\eta(0<\eta<1)$, there exists a constant $C$ which is independent of $n$ and $K(n)$ such that

$$
\begin{aligned}
& \log M\left(\Omega\left(-\sigma, \sigma,\left|z_{n}\right|^{1-\eta},\left|z_{n}\right|\right), f\right) \\
& \leq C\left|z_{n}\right|^{(\pi / \sigma) \eta}\left\{\left(\log \left|z_{n}\right|+\log K(n)+1\right)\right\} .
\end{aligned}
$$

Proof. It suffices to prove the conclusion holds when $n$ is sufficiently large. Consider the transformation

$$
\tau(z)=\frac{z^{\pi /(4 \sigma)}-\left|z_{n}\right|^{\pi /(4 \sigma)}}{z^{\pi /(4 \sigma)}+\left|z_{n}\right|^{\pi /(4 \sigma)}} .
$$

It is easy to see that under this transformation $z=\left|z_{n}\right|$ is mapped to $\tau=0$, and $\Omega(-2 \sigma, 2 \sigma)$ is mapped to the disc $|\tau|<1$. Furthermore $\Omega\left(-\sigma, \sigma,\left|z_{n}\right|^{1-\eta},\left|z_{n}\right|\right)$ is mapped into $|\tau|<t$, where $t=1-(1 / 4)\left|z_{n}\right|^{-(\pi /(2 \sigma)) \eta}$.

Since $f(z)$ assumes $\alpha$ and $\beta$ only at finite number of points on $\Omega(-2 \sigma, 2 \sigma)$, 
hence when $n$ is large enough, these points are mapped outside of the disc $|\tau|<$ $(1+t) / 2$. Set

$$
g(\tau)=\frac{f(z(\tau))-\alpha}{\beta-\alpha},
$$

where $z(\tau)$ is the inverse function of $\tau(z)$. Then $g(\tau)$ dose not assume $0,1, \infty$ on $|\tau|<(1+t) / 2$. When taking $R=(3+t) / 4, r=(1+t) / 2$ and $d=(1+t) / 2$ in Lemma 2 we deduce that

$$
T\left(\frac{1+t}{2}, g\right) \leq \frac{C}{1-t}\left\{\log \frac{1}{1-t}+\log ^{+}|g(0)|+1\right\},
$$

hence

$$
\begin{aligned}
\log M(t, g) & \leq \frac{(1+t) / 2+t}{(1+t) / 2-t} T\left(\frac{1+t}{2}, g\right) \\
& \leq \frac{C}{(1-t)^{2}}\left\{\log \frac{1}{1-t}+\log ^{+}|g(0)|+1\right\} .
\end{aligned}
$$

Since $1-t=(1 / 4)\left|z_{n}\right|^{-(\pi /(2 \sigma)) \eta}$ and $g(0)=\left(f\left(\left|z_{n}\right|\right)-\alpha\right) /(\beta-\alpha)$ we have

$\log M\left(\Omega\left(-\sigma, \sigma,\left|z_{n}\right|^{1-\eta},\left|z_{n}\right|\right), f\right) \leq \log M(t, g)+C$

$$
\leq C\left\{\left|z_{n}\right|^{(\pi / \sigma) \eta}\left(\log \left|z_{n}\right|+\log K(n)+1\right)\right\} .
$$

\section{The proof of Theorem 1}

The proof of Theorem 1 .

Without loss of generality we assume that $\theta=0$, i.e., we consider the ray $\chi(0)=\{z: \arg z=0\}$. If $\chi(0)$ is not a Julia direction of $f(z)$, then there exists a sector $\Omega(-2 \sigma, 2 \sigma)$ and two distinct complex numbers $\alpha, \beta$ such that $f(z)$ assumes $\alpha$ and $\beta$ only finite number of times. Now we prove that on the sector $\Omega(-\sigma / 128, \sigma / 128)$,

$$
\lim _{\substack{|z| \rightarrow+\infty \\ z \in G}} \frac{\log \log |f(z)|}{\log |z|}=\lambda
$$

holds.

If the conclusion of Theorem 1 were not true, then there exists a positive number $\rho(0<\rho<\lambda)$ and a sequence $\left\{z_{n}\right\} \quad\left(\left|z_{n}\right| \rightarrow \infty\right)$ in $\Omega(-\sigma / 128, \sigma / 128)$ such that

$$
\log \left|f\left(z_{n}\right)\right| \leq\left|z_{n}\right|^{\rho} .
$$

Hence from Lemma 3 we have

$$
\log \left|f\left(\left|z_{n}\right|\right)\right| \leq C\left\{\left|z_{n}\right|^{\rho}+1\right\} .
$$


Take a positive number $\eta$ such that $(\pi / \sigma) \eta+\rho<(1-3 \eta) \lambda$, from Lemma 4 we can deduce that

$$
\begin{aligned}
& \log M\left(\Omega\left(-\sigma, \sigma,\left|z_{n}\right|^{1-\eta},\left|z_{n}\right|\right), f\right) \\
& \leq C\left\{\left|z_{n}\right|^{(\pi / \sigma) \eta}\left(\log \left|z_{n}\right|+\left|z_{n}\right|^{\rho}+1\right)\right\} \\
& \leq\left|z_{n}\right|^{\lambda(1-2 \eta)}
\end{aligned}
$$

holds for $n$ is sufficiently large.

By Lemma 1 there exists an asymptotic path $L$ tending to $\infty$, such that for any $\varepsilon>0(\varepsilon<\lambda-\lambda(1-2 \eta) /(1-\eta))$,

$$
\log |f(z)| \geq|z|^{\lambda-\varepsilon}, \quad z \in L
$$

holds for $|z|$ is sufficiently large. Hence

$$
\log |f(z)| \geq\left|z_{n}\right|^{(\lambda-\varepsilon)(1-\eta)}
$$

holds for $z \in L$ and $\left|z_{n}\right|^{1-\eta} \leq|z| \leq\left|z_{n}\right|$.

Obviously, there exists an positive number $N^{\prime}$ in the interval $\left[(1 / 4)\left|z_{n}\right|^{(1-\eta)(\lambda-\varepsilon)},(1 / 2)\left|z_{n}\right|^{(1-\eta)(\lambda-\varepsilon)}\right]$ such that $f^{\prime}(z)$ has no zero points on the level set $L_{n}=\left\{z: \log |f(z)|=N^{\prime}\right\}$, hence $L_{n}$ consists of analytic curves. Consider the set $F_{n}=\left\{z: \log |f(z)|>N^{\prime}\right.$ and $\left.|z|<\left|z_{n}\right|\right\}$. Let $z_{n}^{\prime}$ be a point at which $L$ intersects the circle $\left\{z: z=\left|z_{n}\right|^{1-\eta}\right\}$, and $\Omega_{n}$ be the connected component of $F_{n}$ that contains $z_{n}^{\prime}$. By maximum modulus principle, $\overline{\Omega_{n}} \cap\left\{z: z=\left|z_{n}\right|\right\} \neq \emptyset$. Let $\theta_{t}$ be the part of $\{z:|z|=t\}$ lying in $\Omega_{n}, \theta(t)$ and $t \theta(t)$ be the angle measure and linear measure of $\theta_{t}$ respectively. Notice that

$$
\left|z_{n}\right|^{(\lambda-\varepsilon)(1-\eta)} \geq\left|z_{n}\right|^{\lambda(1-2 \eta)} \text {. }
$$

Hence from (1) and (2) we have

$$
1=\theta(t) \frac{1}{\theta(t)} \leq(2 \pi-2 \sigma) \frac{1}{\theta(t)}, \quad\left|z_{n}\right|^{1-\eta} \leq t \leq\left|z_{n}\right|,
$$

Let $\omega\left(z, \theta_{t}\right)$ be the harmonic measure of $\theta_{t}$ with respect to $F_{n}$. From $[3,111-$ 117] we have the estimate

$$
\omega\left(z_{n}^{\prime}, \theta_{t}\right) \leq 9 \sqrt{2} \exp \left\{-\pi \int_{2\left|z_{n}\right|^{1-\eta}}^{(1 / 2)\left|z_{n}\right|} \frac{d t}{t \theta(t)}\right\} .
$$

Hence, by the maximum principle we have

$$
\log \left|f\left(z_{n}^{\prime}\right)\right| \leq N^{\prime}+9 \sqrt{2} \exp \left\{-\pi \int_{2\left|z_{n}\right|}^{(1 / 2)\left|z_{n}\right|} \frac{d t}{t \theta(t)}\right\} \log M\left(\left|z_{n}\right|, f\right) .
$$

Noticing that $N^{\prime} \in\left[(1 / 4)\left|z_{n}\right|^{(1-\eta)(\lambda-\varepsilon)},(1 / 2)\left|z_{n}\right|^{(1-\eta)(\lambda-\varepsilon)}\right]$, from (2) we have

$$
\left|z_{n}\right|^{(1-\eta)(\lambda-\varepsilon)} \leq 18 \sqrt{2} \exp \left\{-\pi \int_{2\left|z_{n}\right|^{1-\eta}}^{(1 / 2)\left|z_{n}\right|} \frac{d t}{t \theta(t)}\right\} \log M\left(\left|z_{n}\right|, f\right) .
$$


Hence from (3) we have

$$
\begin{aligned}
\frac{\pi}{2 \pi-2 \sigma} \int_{2\left|z_{n}\right|^{1-\eta}}^{(1 / 2)\left|z_{n}\right|} \frac{d t}{t} & \leq \pi \int_{2\left|z_{n}\right|^{1-\eta}}^{(1 / 2)\left|z_{n}\right|} \frac{d t}{t \theta(t)} \\
& \leq \log \log M\left(\left|z_{n}\right|, f\right)-\log \left|z_{n}\right|(1-\eta)(\lambda-\varepsilon)+\log (18 \sqrt{2})
\end{aligned}
$$

i.e.,

$$
\begin{aligned}
\frac{\pi \eta}{2 \pi-2 \sigma} \log \left|z_{n}\right| \leq & \log \log M\left(\left|z_{n}\right|, f\right)-\log \left|z_{n}\right|(1-\eta)(\lambda-\varepsilon) \\
& +\log (18 \sqrt{2})+\frac{\pi}{2 \pi-2 \sigma} \log 4 .
\end{aligned}
$$

Therefore

$$
\begin{aligned}
\frac{\pi \eta}{2 \pi-2 \sigma} & \leq \limsup _{n \rightarrow \infty} \frac{\log \log M\left(\left|z_{n}\right|, f\right)}{\log \left|z_{n}\right|}-(1-\eta)(\lambda-\varepsilon) \\
& \leq \lambda-(1-\eta)(\lambda-\varepsilon) .
\end{aligned}
$$

Let $\varepsilon \rightarrow 0$ we have $\pi \eta /(2 \pi-2 \sigma) \leq \lambda \eta$. Hence

$$
\frac{1}{2}<\frac{\pi}{2 \pi-2 \sigma} \leq \lambda .
$$

This contradicts to that the order of $f(z)$ is less than or equal to $1 / 2$.

The proof of Theorem 1 is completed.

In [2] H. Yoshida proved that, if an entire function $f(z)$ satisfies $\log M(2 r, f) \sim \log M(r, f)$, then the set of ray $\chi(\theta)$ for which $\theta$ is a limit point of the set

$$
E(0, f)=\left\{\arg z_{n}: f\left(z_{n}\right)=0\right\}
$$

is precisely the set of Julia directions of $f(z)$. In this situation, the order $f(z)$ is 0 (see [4]). It is well known that this conclusion does not hold for general entire functions. But we have

COROLlary 1. Let $f(z)$ be an entire function of order $0<\lambda \leq 1 / 2$, and $f(z)$ be regular growth. $\alpha$ is a complex number. Then for any limit point $\theta$ of the set

$$
E(\alpha, f)=\left\{\arg z_{n}: f\left(z_{n}\right)=\alpha\right\},
$$

the ray $\chi(\theta)$ is a Julia direction of $f(z)$. Furthermore, if $\alpha$ and $\beta$ are distinct complex numbers, then the set of ray $\chi(\theta)$ for which $\theta$ is the limit point of the set

$$
E(\alpha, \beta, f)=\left\{\arg z_{n}: f\left(z_{n}\right)=\alpha \text { or } f\left(z_{n}\right)=\beta\right\}
$$

is precisely the set of Julia directions of $f(z)$.

Proof. Let $\theta$ be a limit point of the set $E(\alpha, f)$. If $\chi(\theta)$ is not a Julia direction of $f(z)$, then from Theorem 1 there exists a sector $G$ containing $\chi(\theta)$ 
such that $|f(z)|$ tends to $+\infty$ as $|z| \rightarrow+\infty$ in $G$. Hence $f(z)$ can not assume $\alpha$ infinite number of times in the sector $G$. This contradicts to that $\theta$ is a limit point of the set $E(\alpha, f)$. Hence $\chi(\theta)$ is a Julia direction of $f(z)$.

Furthermore, if $\theta$ is a limit point of the set $E(\alpha, \beta, f)$, by the same discussion as above we can see that $\chi(\theta)$ is a Julia direction of $f(z)$. If $\theta$ is not a limit point of the set $E(\alpha, \beta, f)$, then there exists a sector $G$ containing $\chi(\theta)$ such that $f(z)$ assumes $\alpha$ and $\beta$ at most finite number of times in the sector $G$. Hence $\chi(\theta)$ is not a Julia direction of $f(z)$.

\section{REFERENCES}

[ 1 ] K.-H. Chang, Asymptotic values of entire and meromorphic functions, Sci. Sinica, 20 (1977), $720-739$.

[2] H. Yoshida, Julia directions of entire functions of smooth growth, Nagoya Math. J., 87 (1982), 41-57.

[ 3 ] M. Tsuj, Potential Theory in Modern Function Theory, Maruzen, Tokyo, 1959.

[4] W. K. Hayman, On Iversen's theorem for meromorphic functions with few poles, Acta Math., 141 (1978), 115-145.

[5] L. YANG, Value Distribution Theory, Springer-Verlag, Berlin-Heidelberg, 1993.

[6] L. YAng, Value Distribution Theory and New Research on it, Science Press, Beijing, 1982 (in Chinese).

Department of Mathematics

FUDAN UNIVERSITY

SHANGHAI 200433

CHINA

e-mail: jinluk@online.sh.cn 